\title{
Qualitative, Quantitative, or Mixed Methods? A Systematic Scoping Review on Methodological Applications in Ageing and Dementia Studies
}

Atiqur sm-Rahman ( $\nabla$ atiqur.rahman@liu.se )

Linköping University https://orcid.org/0000-0002-7675-2415

Yasmin Jahan

Hiroshima University Faculty of Medicine Graduate School of Biomedical and Health Sciences:

Hiroshima Daigaku Igakubu Daigakuin Ishiyaku Hokengaku Kenkyuka

\section{Research}

Keywords: ageism, dementia, PRISMA-ScR, qualitative study, quantitative study, mixed-methods, review.

Posted Date: October 1st, 2020

DOl: https://doi.org/10.21203/rs.3.rs-77451/v1

License: (c) (i) This work is licensed under a Creative Commons Attribution 4.0 International License.

Read Full License 


\section{Abstract}

Background: Older adults (with and without dementia) are discriminated against at different social levels and often over-generalized in a stereotypical manner is called ageism. Despite advancements in gerontological research, the methodological implementations in the field of ageing and dementia studies have not been shared equally. This article reports on a systematic scoping review of the emerging methodological trends (use and application of research designs and methods) in this combined field.

Methods: The study reviewed evidence-based articles published from 2009 to 2018 and indexed in five scientific electronic databases Web of Science, PubMed, Scopus, PsycINFO, and CINAHL complete by following PRISMA-ScR protocol. Both visual scanning of reference lists and hand searching of leading journals were performed in the field of ageing and dementia in order to maximize the search result.

Results: A total of 112 papers were included. The review reveals that the predominant form of methodological application was a quantitative design (74.1\%) compared to a qualitative $(19.6 \%)$ and mixed methods (6.2\%) in the combined field of ageing and dementia. Furthermore, the data collection instruments mostly used a variety of questionnaire surveys (with and without validated scales) and interviews. Both quantitative, qualitative, and mixed-methods studies have targeted the general public and healthcare professionals by and large, not older adults or people with dementia in particular.

Conclusions: The results have important implications for the methodological advancement of ageing and dementia research, as well as for the development of inter-disciplinary and cross-cultural interventions considering the potentialities and limitations of data collection tools. The study provides a first step towards understanding key aspects in any study setting and recommends to be purposeful about what information will be gathered, which measurement tool or instrument is consistent with study purpose, and how the knowledge will be utilized.

\section{Background}

Globally, the share of the older adults is increasing along with a prevalence of age-associated diseases, such as dementia [1]; and age-based discrimination- called ageism-is prevalent towards this group of people [2,3]. Older adults aged 65 years and over diagnosed with dementia are at higher risk of experiencing ageism because of being older and their diagnosis [4]. This two-fold tagging poses older adults (with and without dementia) into the feelings of loneliness [5] as well as excludes them from meaningful roles in society [6].

The diversity of such challenges could be investigated and explored through empirical studies that are methodologically robust. A compact methodological layout consistent with study purpose is pivotal. It is also advantageous for researchers to enrich knowledge, contribute to theory development, and practical applications of findings with a solid scientific structure [7-10]. Unfortunately, existing studies that examine various aspects of ageing and dementia suffer to an extent from methodological limitations regarding research design, data collection tools, and measurement scales [11, 12]. Moreover, evidence 
suggests that the overall methodological progress in this combined research field has not been as rapid as other disciplines $[13,14]$.

In order to measure and improve the current status of older adults (with and without dementia), the evidence-based gerontological research has used various research design- quantitative [15], qualitative [16], and mixed methods [17] across countries and contexts [3, 18, 19]. A wide range of literature reviews and meta-analysis has published in ageing and dementia studies mainly assessed the study findings and quality of the study. For example, literature review in ageing research, indeed, have highlighted to display different facets of ageism in various health and social care settings [20-26]. Some scholarly reviews on dementia studies examined how negative stereotype, beliefs, and attitudes impact people living with dementia (PIwD) [27-32].

Despite an increasing number of empirical evidence both on aging and dementia research (e.g., [33-36], the field lacks methodological best practices of research design, data collection methods, and assessment tools. A limited number of review studies have argued explicitly for establishing the importance of methodological development in this combined research field [37, 38].

\section{Study Objectives}

Given the context, we conducted a systematic and comprehensive literature search aimed at highlighting emerging methodological trends (use and application) in the combined research field of ageing and dementia. In particular, the study focuses on reviewing the methodology (research design) and methods (data collection tools, measurement scales) in order to identify available methodological practices and mapped the overall state-of-the-art in this research field by synthesizing data from the literature. We considered assessing the diverse research designs that are applied to describe or to identify ageism and/or dementia-related aspects. The scope of this review can serve as an important reference point for the identification of methodological strategies.

\section{Rationale}

The reason to choose this topic underlies the recent forecast where a significant increase of research is being expected on ageing and dementia care in the coming years due to the rapid population ageing [18]. Thus, the current review is timely and has importance especially from the methodological aspects. We present a comprehensive analysis of qualitative, quantitative, and mixed methods studies.

\section{Methods}

The current study follows the PRISMA-ScR (PRISMA extension for Scoping Reviews) search protocol [39]. This approach was chosen because it allows reviewers to map evidence on a topic and identify main concepts, sources, and knowledge gaps that need to be explored. The review was guided by a systematic search and critical review approach that incorporates multiple study types rather than focusing on single preferred study design [40]. Studies focused on ageing (e.g. ageism, discrimination, stereotypes, 
attitudes) and dementia (Alzheimer's disease) related aspects were considered in order to get a comprehensive picture. However, it would be difficult to draw any firm conclusions of the entire research field in a single review.

\section{Search strategy and selection criteria}

Systematic searching was completed in October 2019 to extract literature published between 2009 and 2018. The primary sources of literature were five scientific electronic databases Web of Science, PubMed, Scopus, PsycINFO, and CINAHL complete by using the advanced search option. Databases were determined with the help of an information specialist in the field of Social Sciences. The search strategy and basic search string were developed in consultation with a librarian as well as an expert scientist in this research field. Following the Boolean operators, the logical grid was applied as ((ageism OR discrimination OR prejudice OR stereotype OR stigma OR attitude) AND (dement* OR Alzheimer*)). The search string was intended to retrieve all possible scientific articles related to ageing and dementia regardless of specific methodology and/or methods. Each electronic database uses the same thesaurus terms. Due to study nature, we restrict the search within specific subject headings includes Social Sciences, Nursing, Psychology, Arts and Humanities, Multidisciplinary, and Health Professions. The authors have conducted the search individually and iteratively in order to maximize the quality and outcome of the search. All the identified citations were combined and recorded into the Endnote software version 9 shared by the reviewers. No limitations were defined regarding age and gender of the study participants, hence the review focus on ageing and dementia related aspects to ensure that no relevant article was missed. Finally, articles were screened on the basis of title, abstract, and full-text screening if they met the eligibility (inclusion) criteria.

\section{Inclusion criteria}

- peer-reviewed empirical study (including quantitative/qualitative/mixed methods study);

- published between 2009 and 2018;

- published in English;

- referred ageing and/or dementia or both as the main topic;

- examined discrimination related to old age (65 years and over);

- excluded conference presentations, letter to editor, correspondence, discussion papers or unpublished dissertations.

\section{Data Extraction}

Data were extracted individually by two investigators. After the final selection of the reviewed articles, all relevant information was extracted from the full-text that includes the following items: author, year of publication, country of research, sample size, study design, participant type, data collection tool, and scales used (see supplemental Table A). The extracted data were enlisted in a data-extraction table using a Microsoft Excel spreadsheet and a pilot-testing was performed on fifteen randomly selected articles to 
ensure the suitability and exhaustiveness of the tool. Any disagreements were resolved by consensus agreement and by a discussion between investigators. It is worthwhile mentioning that we intentionally do not perform quality assessment [41] and avoided discussing the findings of review studies that will be presented elsewhere. As this is a methodological review article, we felt that including such discussion, even briefly, would be more distracting than informative.

\section{Data analysis}

The selected articles were valued against the same underlying criteria and were grouped into two basic categories: methodological approach, and data collection instruments. First, we developed a descriptive summary of the studies and examined based on the basic categories; and then used descriptive statistical analysis. The geographic location of the reviewed studies was categorization followed by the World Bank's country classification-2020 [42].

\section{Results}

\section{Search results}

The database search produced a total of 6,979 records, of those 3372 duplicates (3088 by Endnote and 284 by hand search) were removed. From the remaining, 3149 were excluded after screening the title and abstract. We identified 458 evidence-based studies for full-text screening where 97 studies met all inclusion criteria. Another 15 articles were added from manual reference mining of leading journals in ageing and dementia field. Finally, 112 articles were subjected to review (see figure 1). It is worth noting that all selected articles and their citation are enlisted based on the study design presented in supplemental Table A.

\section{Description of Included Studies}

A total of $n=22(19.6 \%)$ studies adhered qualitative approach as study design, and the data collection techniques followed by traditional qualitative methods such as interviews, focus group discussion (FGD), case study. Almost three-quarters of the total studies followed a quantitative design $(n=83,74.1 \%)$ and employed different questionnaire survey tools and scales for data collection. Mixed methods studies $(n=7,6.2 \%)$ were the least adopted approach among selected studies, and they applied different quantitative (e.g. questionnaire survey) and qualitative (e.g. interviews) tools in order to gather field data. The methodological distribution of the reviewed studies is displayed in figure 2 .

We found the included research items were conducted in 33 different countries in all five continents of the world. The geographic allocation of the studies was higher in developed or high-income countries compared to other parts of the globe. A total of $n=100$ (89.3\%) studies conducted in high-income regions (Europe $n=40$, North America $n=36$, East Asia $n=8$, Middle East $n=8$, and Oceania $n=8)$. Only $n=12(10.7 \%)$ studies were undertaken in developing $(n=8,7.1 \%)$ and lower- and middle-income countries $(n=4,3.6 \%)$. 
Most of the reviewed articles were published in the second half of the selected timeframe (2014-2018, $n=67,60 \%)$ and the highest number of studies conducted in $2017(n=19,17 \%)$. The cumulative number of publications increased consistently during the subsequent years from 2009.

The organization of the review results is clustered into three methodological groups: qualitative, quantitative, and mixed methods; and presented accordingly.

\section{Qualitative approach}

The studies under this approach (article number 1-22 in Supplemental Table A) were heavily favouring the classic qualitative data collection methods. While many of the studies used interviews $(n=11,50 \%)$, or FGDs ( $n=4,18.8 \%)$, five studies referred to other qualitative methods which includes the case study $(n=1$, $4.5 \%)$, inductive framing $(n=1 ; 4.5 \%)$, qualitative textual analysis $(n=1,4.5 \%)$, and open-ended questionnaire $(n=2 ; 9 \%)$. The remaining studies $(n=2 ; 9 \%)$ employed both interviews and FGD as the primary data collection method. Nine interview-based studies applied any of two interview methods: semi-structured interviews $(n=6,27.7 \%)$ or in-depth interviews $(n=3,13.6 \%)$ whereas only one study $(4.5 \%)$ covered both.

The qualitative studies followed a number of data analysis tools mainly includes thematic analysis, content analysis, and hermeneutic analysis to present their study findings. It is important to clarify that some $(n=4,18.2 \%)$ were unclear while reporting the data collection and data analysis process. We also found an incompatibility with respect to the type of study participants. While the majority of the studies recruited members of care staff $(n=15,68.1 \%)$ or the general public $(n=5,22.7 \%)$, only two studies $(9 \%)$ considered PIwD as study participants. The sample size in these studies ranged from 7 to 49 (avg. 18) individuals. A couple of studies did not report the study sample $(n=2,9 \%)$ at all. All qualitative studies, broadly, focused on investigating the stigmatic beliefs of, attitudes towards, and perceptions on ageing and dementia.

\section{Quantitative approach}

The largest number of the included studies was quantitative in nature (article number 23-105 in supplemental Table $A$ ) where almost all of them used the traditional questionnaire surveys $(n=81,97.6 \%)$. A minimal number of studies $(n=2,2.4 \%)$ reported their data collection strategy very poorly. The questionnaire survey technique was followed by two different categories: (a) with validated scales $(n=45$, $54.2 \%)$; and (b) without validated scales ( $n=38,45.8 \%)$. It is worthwhile to mention that the two studies did not report any scales in their methodology that were included in the second category.

A wide range of scales was applied in the first category with a partial modification where needed. Nearly half of the studies ( $n=22,48.9 \%)$ employed a single scale. Although the use of two $(n=15,33.33 \%)$ or three $(n=6,13.33 \%)$ scales was common, few studies administered five $(n=1,2.22 \%)$ and seven $(n=1$, $2.22 \%)$ scales at a time. On the other hand, studies belong to the second category also demonstrated various scopes of use of different survey techniques. While the most frequently used data collection tool 
was in-person questionnaire survey $(n=25,65.8 \%)$, a couple of studies $(n=8,21.0 \%)$ adopted an online platform. Besides that, very few studies choose a telephone survey $(n=2,5.3 \%)$, postal questionnaire survey $(n=2,5.3 \%)$, and key informant survey $(n=1,2.6 \%)$ as a means of data collection. Further, we found the vignette methodology in five studies. Three of them owned by the first category and the remaining two studies belonged to the second category.

We found a considerable variation in the application of statistical data analysis. The descriptive statistics were more common than other types of analysis namely logistic regression analysis, confirmatory factor analysis, univariate or multivariate analysis. The highest number of studies targeted the general public $(n=44,53.0 \%)$ and a good number of studies recruited the members of care staff $(n=27,32.5 \%)$. Some study also selected informal caregivers $(n=6,7.2 \%)$, older adults $(n=3,3.6 \%)$, and PIwD and their family caregivers $(n=3,3.6 \%)$ as study participants. The sample size in reviewed studies ranged from a minimum of 20 to a maximum of 7669 (avg. 633.4). One study did not report the samples whereas three studies ended up with an average of 21 samples. The research purposes, in general, were to measure the knowledge, attitudes, and beliefs towards ageing and dementia.

\section{Mixed methods approach}

The studies adopted a mixed-methods approach (article number 106-112 in supplemental Table A) mainly relied on FGDs and structured survey questionnaires for the qualitative and quantitative part, respectively. In the qualitative part, the FGD technique was adopted by more studies ( $n=5,71.4 \%)$ than interviews ( $n=2,28.6 \%)$. Only one study (14.3\%) used both case studies and FGDs. All seven studies applied different types of questionnaire survey techniques in order to collect quantitative data. With respect to validated scales, more studies applied a single scale $(n=3,42.9 \%)$. Remaining studies $(n=2$, $28.6 \%)$ applied a researcher-developed survey questionnaire.

Further, the data analysis procedure, study participants, and sample size varied across studies. Even though all seven studies mentioned their scientific or statistical data analysis software, only a couple of studies ( $n=2,28.6 \%)$ had a clearer description of the data analysis procedure. The participants in mixedmethods studies included mainly the general public $(n=4,57.1 \%)$, and various healthcare staff ( 3 out of 7 , 42.9\%). Besides that, in general, the sample size in these studies ranged from 11 to 313 (supplemental Table A). While some studies ( $n=4,57.1 \%)$ precisely distinguished the study participants for both qualitative and quantitative part, the remaining $(n=3,42.6 \%)$ were reluctant to this regard. Due to unspecific sample size distribution, the last three studies made it difficult to draw a precise conclusion. The overall study topics fall mostly into the beliefs of and attitudes towards ageing process and dementia.

\section{Discussion}

The findings of this review contribute to the general body of ageing and dementia research. It intends to clarify two main questions: What is the general tendency of methodological interest in ageing and dementia research? and How diverse is the data collection tools in this research field? Reviewed studies 
offer different methodological approaches to potentially yield quality information on diverse aspects in ageing and dementia research. The key findings have confirmed that one methodological approach (quantitative) is used to a greater extent than other approaches (qualitative, and mixed methods). Many studies notably achieved required methodological criteria such as clearer study purpose, theoretical standpoint, literature review, sampling procedure, data collection methods, and data analysis procedure. However, some studies have failed to fulfill several methodological rigours than is recommended [43-45]. Even though a wide array of methods was used in all approaches, our critical analysis identified FGD, interview, and questionnaire survey technique as the most predominant that guided this research filed. The review results further highlighted areas where qualitative, quantitative, and mixed methods approach can provide clearer guidance.

The following sections are focused on the potentiality of different methodological practices and methodological considerations in ageing and dementia research. Finally, we placed some thoughtprovoking recommendations based on our review insights that might guide formulating future research design in this field.

\section{Potentialities of different methodological practice in ageing and dementia}

\section{Qualitative studies}

The reviewed qualitative studies captured the lived experiences and perceived beliefs of various participants- older adults, PlwD, the general public, health care providers, and caregivers- within their own social context. Differences in perspectives assisted to explore why cultural factors are important and how the caregivers respond to it. For example, traditional caregiving practice has identified the influence of cultural perceptions where the use of language played a crucial role. In addition, the perspectives of caregivers showing how their response to ageing and dementia shapes older adults' (with/without dementia) help-seeking and coping strategies. Similarly, the studies aimed at exploring stigma related to ageing and dementia found a relation between social perceptions of dementia and the cultural beliefs of ageing in a given society. The qualitative findings have uncovered some areas that may otherwise be overlooked in ageing and dementia research.

However, the reliance on a single data collection method in most studies, except two studies [46, 47], has restricted the wide scope of the research field. These studies could benefit from using multiple data collection methods while recommending to future research. This finding supports the claim that using a variety of qualitative methods in researching older adults' (with/without dementia) perceptions and experiences is a valuable approach [48].

Equally, striking in this review was that a good number of studies depended on relatively small sample size [49-54]. Some of them described sample estimation procedure very loosely where the determination of data saturation (e.g. time and reason for ceasing data collection) was unclear [53, 54]. In addition, the lack of reflexivity, dubious data interpretation, and incomplete transparency of analytic processes made some of the studies difficult to draw a precise conclusion. These studies were more likely to less 
consistent between their research question(s) and methodology. A resultant concern is that if the steps of methodologic implementation and data analysis procedure is not sufficiently stated, the study findings may not be achieved data comprehensiveness and trustworthiness [55]. So, the methodological principles in qualitative research need to be clearly articulated considering the purposes of research [56].

\section{Quantitative studies}

The abundance of quantitative approach in reviewed studies delineated its explicit popularity in ageing and dementia research. Findings reported in quantitative studies have provided valuable insight into various aspects of the research field. The studies applied different survey techniques, for example, underlined the importance of public attitudes and stigmatic beliefs related to ageing and dementia [57, 58]. However, the key role of cultural backgrounds that facilitates or obstructs the beliefs and attitudes in society was not clearly defined [59].

Our critical analysis further revealed that the conclusive remarks in many of the considered studies demonstrated aggregation bias [60]. That is, the study results often reflect the expectations of the researchers (/authors). Probably that the reason a substantial number of studies have used crosssectional design [61-63]. The study design has recently been criticized in terms of the value of the advancement of aging theory [64]. Study also argued that cross-sectional design might not be appropriate as age-related characteristics are nonlinear and change over time [65]. Therefore, it is suggested combining 'between-person differences' and 'within-person rates of change' while designing a quantitative study in order to maintain methodological comprehensiveness [66].

Studies implemented validated scales either have evaluated the outcomes or determined the effects of interventions. Despite adequate structural validity and internal consistency in some scales (e.g. ADKS, $A D Q, S I S)$, many other scales (e.g. CDR, FSADS, PRISM-PC) may benefit from revisions to improve their content validity. It is worth noting that studies aimed at measuring 'perceptions' or 'attitudes' in ageing research might be inconclusive if other associated dimensions (e.g. stereotypes and prejudice) are overlooked. The intended contextual meanings are important to get a better understanding of such a concept.

Moreover, this review found a majority of implemented scales had an intention to measure the degree to which the expected effects have been achieved $[67,68]$. It can be, thus, suggested that the scales should have a cross-cultural validation, criterion validity, and responsiveness to the scale prior to the final implementation [69]. The challenges can also be compensated through a sound knowledge of the basic principles of measurement scales [68, 70]; and greater usage of qualitative techniques (in particular, ethnography and unstructured interviews) could be advantageous.

Quite a few quantitative studies suffer from the reliability and accuracy of survey data, interpersonal incomparability of survey responses, and the risk of measurement errors. To deal with this problem, a couple of studies anchored the vignette method as a data collection tool apart from the traditional survey practice. Even though vignettes have an advantage for reducing the incomparability of survey results, the 
method might not be applicable when applying with older adults or PIwD [71]. One considerable reason is the heterogeneity among older adults (with/without dementia) who often encounter challenges related to physical and cognitive functions.

Incorporation of diverse groups of individuals (e.g. older adults and PIWD, the general public, care staff, students, and informal caregivers) strengthen many of the quantitative findings where the representative sample size was an issue. Studies lacking a representative sample size may be at risk of presenting a less than optimal product and lessen its impact. In fact, a representative sample is crucial for research on ageing and dementia in order to test hypotheses accurately and draw valid conclusions [33].

Similarly, despite the affluent use of descriptive statistics and logistic regression analysis, a considerable number of studies poorly disclosed how potential problems with missing data were assessed. While it is nearly impossible to achieve complete data capture, quantitative studies can be benefitted from using common missing data methods to avoid incomplete conclusions [37, 72, 73].

\section{Mixed Methods Studies}

Only the quantitative component appears to have been reported in the reviewed mixed methods studies. Thus, the findings from one strand (quantitative) make the study outcome difficult to bring that into meaningful contact. One study, for example, was firm in describing the methodological process whereas the result predominantly reported only quantitative data, and the discussion was ended up without specific direction [74]. Another study, similarly, suffered from a number of limitations such as missing values of variables, non-representative survey sample, considering only a specific type of participants [75]. Findings from such studies make it difficult for other researchers who want to replicate the study design or to implement the study instrument in different contexts.

\section{Methodological considerations in ageing and dementia research}

If age and dementia-related stigma are modeled as dependent on ageing process or diagnosis of dementia, it might be possible to measure the association or correlation between stigma and quality of life outcomes using a structured questionnaire or validated scale [76]. On the other hand, if stigma is investigated using the social constructionist model [77], a qualitative approach can be useful to explore how the participants' perceptions are mapped onto conceptualizations of stigma; and to examine the interactions between the older adults or PIWD and other individuals [78]. These are just two examples to understand how a particular approach might guide or place restrictions to explore or measure the studied phenomenon.

\section{Qualitative approach}

A qualitative approach has the potential to offer an in-depth view as well as a description of factors that facilitate or impede the associated social perceptions of older adults (with/without dementia) $[79,80]$. A growing body of literature has found the method useful in eliciting individual's beliefs, values and attitudes, behavioral practice, meaning and process of ageing and perceptions on dementia [81-85]. This 
approach can explore the heterogeneous characteristics of older adults where testing a hypothesis might not be a suitable attempt to capture these conceptual phenomena $[79,86]$. However, the qualitative methods would not be appropriate if the study's purpose does not match with the research approach [87].

\section{Quantitative approach}

The purposes of a quantitative approach are to obtain and analyse numerical data to describe, explain, predict, control, or measure the causal interrelationships among variables and phenomenon of interest $[88,89]$. This approach is convincing in ageing and dementia research in order to describe the reason for and the extent to which older adults (with/without dementia) differ from or similar to each other [90]. A cross-sectional study, for example, is useful to evaluate the individual differences in the health-related needs of older adults from a single point of time. The findings may, therefore, generate a hypothesis that eventually longitudinal study could test. However, the result might be limited to making causal inference [91]. In addition, it is argued that the quantitative approach reduces behavioral practices to numerical data may not capture the complexity of ageing population and PIwD [90,92]. Considering the merits and limits, it is important to remember that the value of quantitative studies depends on the choice of appropriate method, the decision about what to measure, and the type of analysis to interpret the findings.

\section{Mixed Methods approach}

A mixed-methods approach has advocated in many ageing and dementia studies with accompanying a range of sequential and concurrent strategies [93-96]. The approach is useful in ageing research due to the nature of including fewer missing data, enhancing validity (internal and external), and combining the benefits of generalizability in a single study [96-100]. However, the wording in question, order of questions, time and resources allocations, sample size determination, and consensus on data analysis procedure are few challenges should take into account throughout the study $[96,101,102]$. Therefore, it is important to take into account whether a mixed-methods study adds any value in understanding and interpretations of ageing- and dementia-related issues compared with a purely quantitative or purely qualitative study.

The entire discussion assists us to conclude that quantitative and qualitative approaches represent diverse aspects of a continuum and are not discrete or polar opposites [103]. A quantitative approach might get a preference to answer a precise clinical research question in a highly specific way by controlling extraneous variables [104]. Nonetheless, to know other associated significant factors such as social influence, quality of life, individuals' perception is equally important. In this case, a qualitative approach could be useful to explore the meanings of the phenomenon as well as engenders new insights through induction from data $[104,105]$. The knowledge acquired from both approaches would allow us to comprehend the real impact of ageing- and dementia-related aspects, and perceptions of the community [1]. A mixed-methods approach, as an alternative, may integrate both qualitative and quantitative evidence in order to provide a more complete understanding of a research problem than either approach 
alone. Therefore, it can be anticipated that evaluating a phenomenon into a single vision can restrict other prospects that are open to research.

\section{Recommendations and Future direction}

This systematic scoping review clearly highlights substantive and methodologic gaps in the literature that could be benefitted from future research. Accordingly, our review identifies a tendency to use a specific research approach with suboptimal engagement, through limited sampling, of older adults (with/without dementia) within studies. Overall, this gap heightens a critical question of how contemporary studies can provide conclusive knowledge of the diversity of older adults (with/without dementia). Thus, we recommend a more purposeful and broader integration of sub-groups of participants. Cross-cultural multidisciplinary studies could be an asset.

Moreover, the traditional research methods in reviewed studies might not be suitable for describing the complexities in the combined field of ageing and dementia [106]. It can be argued that methodological approaches and tools do not need any drastic change; hence, the inclusion of interdisciplinary tools could merit in future research in this field. The base of these recommendations is derived from the understanding of the relative absence of theoretical articulation with implemented methods in the reviewed studies.

The review result, further, suggests developing a more thorough design in a study to capture a wide variety (not only 'structural' but also 'processual' aspects) of the phenomenon that therefore widens and enlarges our notions. Besides that, a methodological tool (e.g. questionnaire, scales, guidelines, checklists) based on the researcher's knowledge might be of benefit for descriptive purposes, could limit scientific value. Therefore, the researcher needs a more riguorous approach to selecting the measurement tools by following validation guidelines and the context in which the tool will be employed. For instance, a specific measure developed for a community setting may not be suitable for use in a clinical setting. Likewise, a validated scale that exclusively developed to examine variations in care needs for older adults (with/without dementia) would allow for comparing typological differences. However, for research that investigates to explore the association between care needs and individuals' attitudes and beliefs, an indepth data collection strategy may be more appropriate. From this perspective, we strongly suggest considering some key aspects in any study setting that are: be purposeful about what information will be gathered, which measurement tool or instrument is consistent with study purpose, and how the knowledge will be utilized.

\section{Limitations Of The Review}

Every attempt of search of the literature was somewhat exhaustive, thereby, it is possible that some relevant studies still be missing unintentionally. We acknowledge that the dominance of quantitative study might have an influence on the overall discussion. In our viewpoint, the selection procedure may suffer from the reviewers' subjective judgements. Adding scientific articles only written in English and peer-reviewed could be recognized as another limitation of the review that would be an important issue to 
discuss further. However, needless to say, the inclusion of three types of study (qualitative, quantitative, and mixed methods) has extended the scope of this review. In addition, the reference mining of leading journals in the combined field of ageing and dementia has complemented the electronic searches. Finally,

the inter-reviewer agreement and the individual full-text screening by the reviewers have strengthened the review process to decrease probable misinterpretations.

\section{Conclusions}

The indeterminate or inconsistent results and the diverse range of methodological implementations reflected in the reviewed studies make the research area rather disparate and somewhat difficult to interpret. At this point, it might not be an exaggeration to say that the researchers from different disciplines have applied various models using specific techniques to examine or describe a phenomenon related to ageing and dementia while ruling out others. This result signals a need to choose useful research design and methods for particular purposes. However, no research design is optimal for all purposes. Typically, it is the research question that leads the researchers to determine data collection methods. We also agree the interdisciplinary practice may not easily be transposed into the diversified filed of ageing and dementia studies. Nevertheless, the incorporation of interdisciplinary instruments might help to clarify some methodological challenges outlined above; and can then be developed and implemented as suitable data collection tools. Otherwise, the growing ageing community along with a heterogeneous group of PlwD would be excluded and positioned outside the boundary of our understanding.

\section{Abbreviations}

A-ADS: Adolescent Attitudes toward Dementia Scale

AARDS: Attitude toward Alzheimer's disease and Related Dementias Scale.

ADAT: Alzheimer's Disease Awareness Test

ADKQ: Alzheimer's Disease Knowledge Questionnaire

ADKS: Alzheimer's Disease Knowledge Scale

ADKT: Alzheimer's Disease Knowledge Test

ADQ: Approaches to Dementia Questionnaire

AED: Attitudes toward the Elderly with Dementia

AQ-9: Attribution Questionnaire-9

ASS: Affiliate Stigma Scale 
ATA: Attitude toward Aging (subscale)

ATHCTS: Attitudes Toward Health Care Teams Scale

CDR: Clinical Dementia Rating Scale

CES: Caring Efficacy Scale

CES-D: Center for Epidemiologic Studies Depression Scale

DAS: The Dementia Attitude Scale

DBD: Dementia Behavior Disturbance Scale

DKQ: Dementia Knowledge Questionnaire

DSSI: Duke Social Support Index (23-item)

FADS: Fear of Alzheimer's Disease Scale

FGD: Focus group discussion

FMTCS: Farren's Finding Meaning Through Caregiving Scale

FSA: Fraboni Scale of Ageism

FS-ADS: Family Stigma in Alzheimer's disease Scale

FSSS: Family Stigma Stress Scale

GDS: Geriatric Depression Scale

HADS: Hospital Anxiety and Depression Scale

IADL: Lawton Instrumental Activities of Daily Living scale

IEQ: Involvement Evaluation Questionnaire

LIA: Language in Adulthood (LIA) Questionnaire

LSNS: Lubben Social Network Scale

MS: Mastery Scale

PIwD: People living with Dementia

PRISM-PC: Perceptions Regarding Investigational Screening for Memory in Primary Care 
RMBPC: Revised Memory and Behavior Problems Checklist

RSAS: Recreational and Social Activities Scale

SCS: Spiritual Coping Strategies scale.

SES: Self-Esteem Scale

SEWDR: Staff Experience of Working with Demented Residents Scale

SEWDRQ: Staff Experience of Working with Residents with Dementia Questionnaire

SIS: Stigma Impact Scale

ZBI: Zarit Burden Interview

ZBI-SF: Zarit Burden Interview Short Form

Note: Cognitive screening tests scale (MMSE) has removed from the list.

\section{Declarations}

\section{Ethics approval and consent to participate}

Not applicable.

Consent for publication

Yes

\section{Availability of data and materials}

Since it is a review based on secondary literature, all data were extracted from scientific databases that are available.

\section{Competing interests}

No potential conflict of interest was reported by the authors.

\section{Funding}

None

\section{Authors' contributions}

Both authors contributed equally 
The authors would like to express gratitude to Prof. Lars-Christer Hydén for his contribution during the early conceptualization of the review. We are also thankful to the post-doctoral researcher George Pavlidis for his valuable comments in the early version of the manuscript. Authors are indebted to the research team at division ageing and social change for their suggestions in the improvement of the article.

\section{Reference}

1. Pérez-Zepeda MU, García-Peña C, Gutiérrez-Robledo LM: The Need for Differentiated Research Methodology in Aging. In: Aging Research-Methodological Issues. edn.: Springer; 2018: 1-9.

2. Organization WH: World report on ageing and health: World Health Organization; 2015.

3. Ayalon L, Tesch-Römer C: Introduction to the section: Ageism-Concept and origins. In: Contemporary perspectives on ageism. edn.: Springer, Cham; 2018: 1-10.

4. Evans SC: Ageism and dementia. In: Contemporary perspectives on ageism. edn.: Springer, Cham; 2018: 263-275.

5. McHugh KE: Three faces of ageism: society, image and place. Ageing Soc 2003, 23(2):165-185.

6. Wethington E, Pillemer K, Principi A: Research in social gerontology: social exclusion of aging adults. In: Social Exclusion. edn.: Springer; 2016: 177-195.

7. Aguinis $\mathrm{H}$, Vandenberg $\mathrm{RJ}$ : An ounce of prevention is worth a pound of cure: Improving research quality before data collection. Annu Rev Organ Psychol Organ Behav 2014, 1(1):569-595.

8. Salthouse TA: Methodological assumptions in cognitive aging research. The handbook of aging and cognition 2000, 2:467-498.

9. Bangerter LR, Griffin JM, Zarit SH, Havyer R: Measuring the needs of family caregivers of people with dementia: An assessment of current methodological strategies and key recommendations. Journal of Applied Gerontology 2019, 38(9):1304-1318.

10. Pulok MH, van Gool K, Hajizadeh M, Allin S, Hall J: Measuring horizontal inequity in healthcare utilisation: A review of methodological developments and debates. The European Journal of Health Economics 2020, 21(2):171-180.

11. Wilson DM, Errasti-Ibarrondo B, Low G: Where are we now in relation to determining the prevalence of ageism in this era of escalating population ageing?Ageing Research Reviews 2019, 51:78-84.

12. Browne C, Shlosberg E: Attachment theory, ageing and dementia: A review of the literature. Aging and Mental Health 2006, 10(2):134-142.

13. Gray K, Evans SC, Griffiths A, Schneider J: Critical reflections on methodological challenge in arts and dementia evaluation and research. Dementia 2018, 17(6):775-784.

14. Ritchie CW, Terrera GM, Quinn TJ: Dementia trials and dementia tribulations: methodological and analytical challenges in dementia research. Alzheimer's research \& therapy 2015, 7(1):31. 
15. Moniz-Cook E, Vernooij-Dassen M, Woods B, Orrell M, Network I: Psychosocial interventions in dementia care research: the INTERDEM manifesto. In.: Taylor \& Francis; 2011.

16. Goulding A: Arts on prescription for older people: Different stakeholder perspectives on the challenges of providing evidence of impact on health outcomes. Journal of Applied Arts \& Health 2014, 5(1):83-107.

17. Pluye P, Hong QN: Combining the power of stories and the power of numbers: mixed methods research and mixed studies reviews. Annual review of public health 2014, 35:29-45.

18. Levy SR, Macdonald JL: Progress on understanding ageism. Journal of Social Issues 2016, 72(1):525.

19. Lion K, Szcześniak D, Bulińska K, Evans S, Evans S, Saibene F, d'Arma A, Farina E, Brooker D, Chattat R: Do People With Dementia Experience Stigma? A Cross Country Comparison Between Italy, Poland and the UK. Aging \& Mental Health 2019.

20. Burnes D, Sheppard C, Henderson Jr CR, Wassel M, Cope R, Barber C, Pillemer K: Interventions to reduce ageism against older adults: a systematic review and meta-analysis. American Journal of Public Health 2019, 109(8):e1-e9.

21. Chang E-S, Kannoth S, Levy S, Wang S-Y, Lee JE, Levy BR: Global reach of ageism on older persons' health: A systematic review. PloS One 2020, 15(1):e0220857.

22. de São José JMS, Amado CAF: On studying ageism in long-term care: a systematic review of the literature. International psychogeriatrics 2017, 29(3):373-387.

23. Lamont RA, Swift HJ, Abrams D: A review and meta-analysis of age-based stereotype threat: Negative stereotypes, not facts, do the damage. Psychology and aging 2015, 30(1):180.

24. Levy BR, Slade MD, Chang E-S, Kannoth S, Wang S-Y: Ageism amplifies cost and prevalence of health conditions. The Gerontologist 2018, 60(1):174-181.

25. McKeown J, Clarke A, Repper J: Life story work in health and social care: systematic literature review. Journal of advanced nursing 2006, 55(2):237-247.

26. São José JMS, Amado CAF, Ilinca S, Buttigieg SC, Taghizadeh Larsson A: Ageism in health care: a systematic review of operational definitions and inductive conceptualizations. The Gerontologist 2019, 59(2):e98-e108.

27. Alarcón M, del Carmen M: A systematic review about ageism and dementia. 2019.

28. Bethell J, Commisso E, Rostad HM, Puts M, Babineau J, Grinbergs-Saull A, Wighton MB, Hammel J, Doyle E, Nadeau S: Patient engagement in research related to dementia: a scoping review. Dementia 2018, 17(8):944-975.

29. Gilbert J, Ward L, Gwinner K: Quality nursing care in dementia specific care units: A scoping review. Dementia 2017, 18(6):2140-2157.

30. Herrmann LK, Welter E, Leverenz J, Lerner AJ, Udelson N, Kanetsky C, Sajatovic M: A systematic review of dementia-related stigma research: can we move the stigma dial? The American Journal of Geriatric Psychiatry 2018, 26(3):316-331. 
31. Lee M, Ryoo JH, Chung M, Anderson JG, Rose K, Williams IC: Effective interventions for depressive symptoms among caregivers of people with dementia: A systematic review and meta-analysis. Dementia 2019:1471301218822640.

32. Nguyen T, Li X: Understanding public-stigma and self-stigma in the context of dementia: A systematic review of the global literature. Dementia 2020, 19(2):148-181.

33. Bohlmann C, Rudolph CW, Zacher H: Methodological recommendations to move research on work and aging forward. Work, Aging and Retirement 2018, 4(3):225-237.

34. Chaudhury $\mathrm{H}$, Cooke $\mathrm{H}$ : Design matters in dementia care: The role of the physical environment in dementia care settings. Excellence in dementia care 2014, 2:144-158.

35. Rudolph CW, Zacher $\mathrm{H}$ : Considering generations from a lifespan developmental perspective. Work, Aging and Retirement 2017, 3(2):113-129.

36. van Hoof J, Kort HS, van Waarde H, Blom MM: Environmental interventions and the design of homes for older adults with dementia: an overview. American Journal of Alzheimer's Disease \& Other Dementias\& 2010, 25(3):202-232.

37. Chandola T, O'Shea S: Innovative approaches to methodological challenges facing ageing cohort studies: Policy briefing note. In.: National Centre for Research Methods; 2019.

38. Ross F, Donovan S, Brearley S, Victor C, Cottee M, Crowther P, Clark E: Involving older people in research: methodological issues. Health \& social care in the community 2005, 13(3):268-275.

39. Tricco AC, Lillie E, Zarin W, O'Brien KK, Colquhoun H, Levac D, Moher D, Peters MD, Horsley T, Weeks L: PRISMA extension for scoping reviews (PRISMA-ScR): checklist and explanation. Annals of internal medicine 2018, 169(7):467-473.

40. Carroll L, Cassidy JD, Peloso P, Garritty C, Giles-Smith L: Systematic search and review procedures: results of the WHO collaborating centre task force on mild traumatic brain injury. Journal of rehabilitation medicine 2004, 36(0):11-14.

41. Arksey H, O'Malley L: Scoping studies: towards a methodological framework. International journal of social research methodology 2005, 8(1):19-32.

42. Bank W: Country classifications by income level: 2019-2020. 2020.

43. Smith B, McGannon KR: Developing rigor in qualitative research: Problems and opportunities within sport and exercise psychology. International review of sport and exercise psychology 2018, 11(1):101-121.

44. Scrutton R, Beames S: Measuring the unmeasurable: Upholding rigor in quantitative studies of personal and social development in outdoor adventure education. Journal of Experiential Education 2015, 38(1):8-25.

45. Tong A, Sainsbury P, Craig J: Consolidated criteria for reporting qualitative research (COREQ): a 32item checklist for interviews and focus groups. International journal for quality in health care 2007, 19(6):349-357. 
46. Kennedy E, Allen B, Hope A, James IA: Christian worship leaders' attitudes and observations of people with dementia. Dementia (London) 2014, 13(5):586-597.

47. O'Sullivan G, Hocking C, Spence D: Dementia: The need for attitudinal change. Dementia (London) 2014, 13(4):483-497.

48. Flick U: The SAGE handbook of qualitative data collection: Sage; 2017.

49. Navab E, Negarandeh R, Peyrovi H, Navab P: Stigma among Iranian family caregivers of patients with Alzheimer's disease: A hermeneutic study. Nurs Health Sci 2013, 15(2):201-206.

50. Stansbury KL, Marshall GL, Harley DA, Nelson N: Rural African American clergy: an exploration of their attitudes and knowledge of Alzheimer's disease. J Gerontol Soc Work 2010, 53(4):352-365.

51. Walmsley BD, McCormack L: Stigma, the medical model and dementia care: Psychological growth in senior health professionals through moral and professional integrity. Dementia (London) 2016, 15(6):1685-1702.

52. Werner P, Goldstein D, Buchbinder E: Subjective experience of family stigma as reported by children of Alzheimer's disease patients. Qual Health Res 2010, 20(2):159-169.

53. Nedlund AC, Nordh J: Constructing citizens: a matter of labeling, imaging and underlying rationales in the case of people with dementia. Crit Policy Stud 2018, 12(3):253-272.

54. Peel E, Taylor H, Harding R: Sociolegal and practice implications of caring for LGBT people with dementia. Nurs Older People 2016, 28(10):26-30.

55. Nicholas DB, Orjasaeter JD, Zwaigenbaum L: Considering methodological accommodation to the diversity of ASD: A realist synthesis review of data collection methods for examining first-person experiences. Review Journal of Autism and Developmental Disorders 2019, 6(2):216-232.

56. Saunders B, Sim J, Kingstone T, Baker S, Waterfield J, Bartlam B, Burroughs H, Jinks C: Saturation in qualitative research: exploring its conceptualization and operationalization. Quality \& quantity 2018, 52(4):1893-1907.

57. Werner $P$, Hess $A$ : Examining courtesy stigma among foreign health care workers caring for persons with Alzheimer's disease: A focus group study. Home Health Care Serv Q 2016, 35(2):69-85.

58. Gove D, Small N, Downs M, Vernooij-Dassen M: General practitioners' perceptions of the stigma of dementia and the role of reciprocity. Dementia (London) 2017, 16(7):948-964.

59. Carrieri D, Peccatori F, Boniolo G: Supporting supportive care in cancer: the ethical importance of promoting a holistic conception of quality of life. Critical reviews in oncology/hematology 2018, 131:90-95.

60. James LR: Aggregation bias in estimates of perceptual agreement. Journal of applied psychology $1982,67(2): 219$.

61. MacDonald SW, Stawski RS: Methodological considerations for the study of adult development and aging. In: Handbook of the psychology of aging. edn.: Elsevier; 2016: 15-40.

62. Jordão M, Ferreira-Santos F, Pinho MS, St Jacques PL: Meta-analysis of aging effects in mind wandering: Methodological and sociodemographic factors. Psychology and aging 2019, 34(4):531. 
63. Power MC, Weuve J, Sharrett AR, Blacker D, Gottesman RF: Statins, cognition, and dementiasystematic review and methodological commentary. Nature Reviews Neurology 2015, 11(4):220.

64. Fuss BG, Dorstyn D, Ward L: Computer-mediated communication and social support among community-dwelling older adults: A systematic review of cross-sectional data. Australasian Journal on Ageing 2019, 38(4):e103-e113.

65. Hofer SM, Sliwinski MJ, Flaherty BP: Understanding ageing: Further commentary on the limitations of cross-sectional designs for ageing research. Gerontology 2002, 48(1):22-29.

66. Kanfer R, Beier ME, Ackerman PL: Goals and motivation related to work in later adulthood: An organizing framework. European Journal of Work and Organizational Psychology 2013, 22(3):253264.

67. Miele F, Neresini F, Boniolo G, Paccagnella O: Supportive care for older people with dementia: socioorganisational implications. Ageing Soc 2020:1-33.

68. Gupta A, Rehman A: Measurement scales used in elderly care: CRC Press; 2017.

69. Ayalon L, Dolberg P, Mikulionienė S, Perek-Białas J, Rapolienė G, Stypinska J, Willińska M, de la Fuente-Núñez V: A systematic review of existing ageism scales. Ageing research reviews 2019, 54:100919.

70. Block L, Mullen S, Thomas G, Kaiser B, Gilmore-Bykovskyi A: Methodological strategies and challenges for evaluating participant views on dementia research recruitment. Innovation in Aging 2019, 3(Supplement_1):S706-S706.

71. Poksinska BB, Cronemyr P: Measuring quality in elderly care: possibilities and limitations of the vignette method. Total Quality Management \& Business Excellence 2017, 28(9-10):1194-1207.

72. MD MDB, Williamson J, Pahor M: Missing-data in epidemiological studies of age-associated cognitive decline. Journal of the American Geriatrics Society 1999, 47(11):1380-1381.

73. Hardy SE, Allore H, Studenski SA: Missing data: a special challenge in aging research. Journal of the American Geriatrics Society 2009, 57(4):722-729.

74. Adebiyi AO, Fagbola MA, Olakehinde O, Ogunniyi A: Enacted and implied stigma for dementia in a community in south-west Nigeria. Psychogeriatrics 2016, 16(4):268-273.

75. O'Sullivan JL, Gellert P, Hesse B, Jordan LM, Moller S, Voigt-Antons JN, Nordheim J: Exploring attitudes of healthcare professionals towards ICT-based interventions for nursing home residents with dementia: a mixed-methods approach. Contemp Nurse 2018, 54(1):13-25.

76. Burgener SC, Buckwalter K, Perkhounkova Y, Liu MF: The effects of perceived stigma on quality of life outcomes in persons with early-stage dementia: Longitudinal findings: Part 2. Dementia 2015, 14(5):609-632.

77. Sabat SR, Harré R: The construction and deconstruction of self in Alzheimer's disease. Ageing Soc 1992, 12(4):443-461.

78. Gove D, Downs M, Vernooij-Dassen M, Small N: Stigma and GPs' perceptions of dementia. Aging \& mental health 2016, 20(4):391-400. 
79. Cobb AK, Forbes S: Qualitative research: what does it have to offer to the gerontologist? The Journals of Gerontology Series A: Biological Sciences and Medical Sciences 2002, 57(4):M197-M202.

80. Jenkins N: Qualitative Research With People Who Have a Diagnosis of Dementia. In: SAGE research methods foundations. edn.: SAGE Publications; 2019.

81. Roller MR, Lavrakas PJ: Applied qualitative research design: A total quality framework approach: Guilford Publications; 2015.

82. Jones RK: The unsolicited diary as a qualitative research tool for advanced research capacity in the field of health and illness. Qualitative Health Research 2000, 10(4):555-567.

83. Phoenix C, Bell SL: Beyond "move more": feeling the rhythms of physical activity in mid and later-life. Social Science \& Medicine 2019, 231:47-54.

84. Tulle E, Phoenix C: Introduction: Rethinking physical activity and sport in later life. In: Physical Activity and Sport in Later Life. edn.: Springer; 2015: 1-8.

85. van Gennip IE, W. Pasman HR, Oosterveld-Vlug MG, Willems DL, Onwuteaka-Philipsen BD: How dementia affects personal dignity: a qualitative study on the perspective of individuals with mild to moderate dementia. Journals of Gerontology Series B: Psychological Sciences and Social Sciences 2016, 71(3):491-501.

86. Phoenix $C$ : Why qualitative research is needed in gerontology and how we can do it better. The Journals of Gerontology: Series B 2018, 73(7):e81-e85.

87. Marshall C, Rossman GB: Designing qualitative research: Sage publications; 2014.

88. Bloomfield J, Fisher MJ: Quantitative research design. Journal of the Australasian Rehabilitation Nurses Association 2019, 22(2):27.

89. Gay LR, Mills GE, Airasian PW: Educational research: Competencies for analysis and applications: Merrill/Pearson; 2009.

90. Browning C, Kendig H, Minichiello V: Research methods in gerontology. In: An introduction to gerontology: A multidisciplinary perspective. edn. Edited by Minichiello V, Alexander L, Jones D: Prentice Hall; 2014: 184-196.

91. Reyes-Morales H, Doubova SV, Pérez-Cuevas R: Health Systems Research in Aging. In: Aging Research-Methodological Issues. edn.: Springer; 2018: 157-174.

92. Fiske K, Delmolino L: Use of discontinuous methods of data collection in behavioral intervention: Guidelines for practitioners. Behavior Analysis in Practice 2012, 5(2):77-81.

93. Au DW, Woo J, Zaidi A: Extending the active ageing index to Hong Kong using a mixed-method approach: Feasibility and initial results. Journal of Population Ageing 2020:1-16.

94. Stirling C, Andrews S, Croft T, Vickers J, Turner P, Robinson A: Measuring dementia carers' unmet need for services-an exploratory mixed method study. BMC Health Services Research 2010, 10(1):122.

95. Robinson AL, Emden CG, Croft TD, Vosper GC, Elder JA, Stirling C, Vickers JC: Mixed methods data collection in dementia research: a "progressive engagement" approach. Journal of Mixed Methods 
Research 2011, 5(4):330-344.

96. Gallo JJ, Joo JH: Mixed Methods in Geriatrics and Gerontology Research. In: Aging ResearchMethodological Issues. edn.: Springer; 2018: 129-142.

97. Vedel I, Akhlaghpour S, Vaghefi I, Bergman H, Lapointe L: Health information technologies in geriatrics and gerontology: a mixed systematic review. Journal of the American Medical Informatics Association 2013, 20(6):1109-1119.

98. Creswell JW, Clark VLP: Designing and conducting mixed methods research: Sage publications; 2017.

99. Denzin NK: The research act: A theoretical introduction to sociological methods: Routledge; 2017.

100. McKim CA: The value of mixed methods research: A mixed methods study. Journal of Mixed Methods Research 2017, 11(2):202-222.

101. Wittink MN, Barg FK, Gallo JJ: Unwritten rules of talking to doctors about depression: integrating qualitative and quantitative methods. The Annals of Family Medicine 2006, 4(4):302-309.

102. Kessel F, Association NAAP: Interdisciplinary Research: Case Studies from Health and Social Science: Case Studies from Health and Social Science: Oxford University Press, USA; 2008.

103. Newman I, Benz CR, Ridenour CS: Qualitative-quantitative research methodology: Exploring the interactive continuum: SIU Press; 1998.

104. Gibson G, Timlin A, Curran S, Wattis J: The scope for qualitative methods in research and clinical trials in dementia. Age and Ageing 2004, 33(4):422-426.

105. Bolderston A: Five Percent Is Not Enough! Why We Need More Qualitative Research in the Medical Radiation Sciences. Journal of medical imaging and radiation sciences 2014, 45(3):201-203.

106. Keady J, Hydén L-C, Johnson A, Swarbrick C: Social research methods in dementia studies: Inclusion and innovation: Routledge; 2017.

\section{Figures}




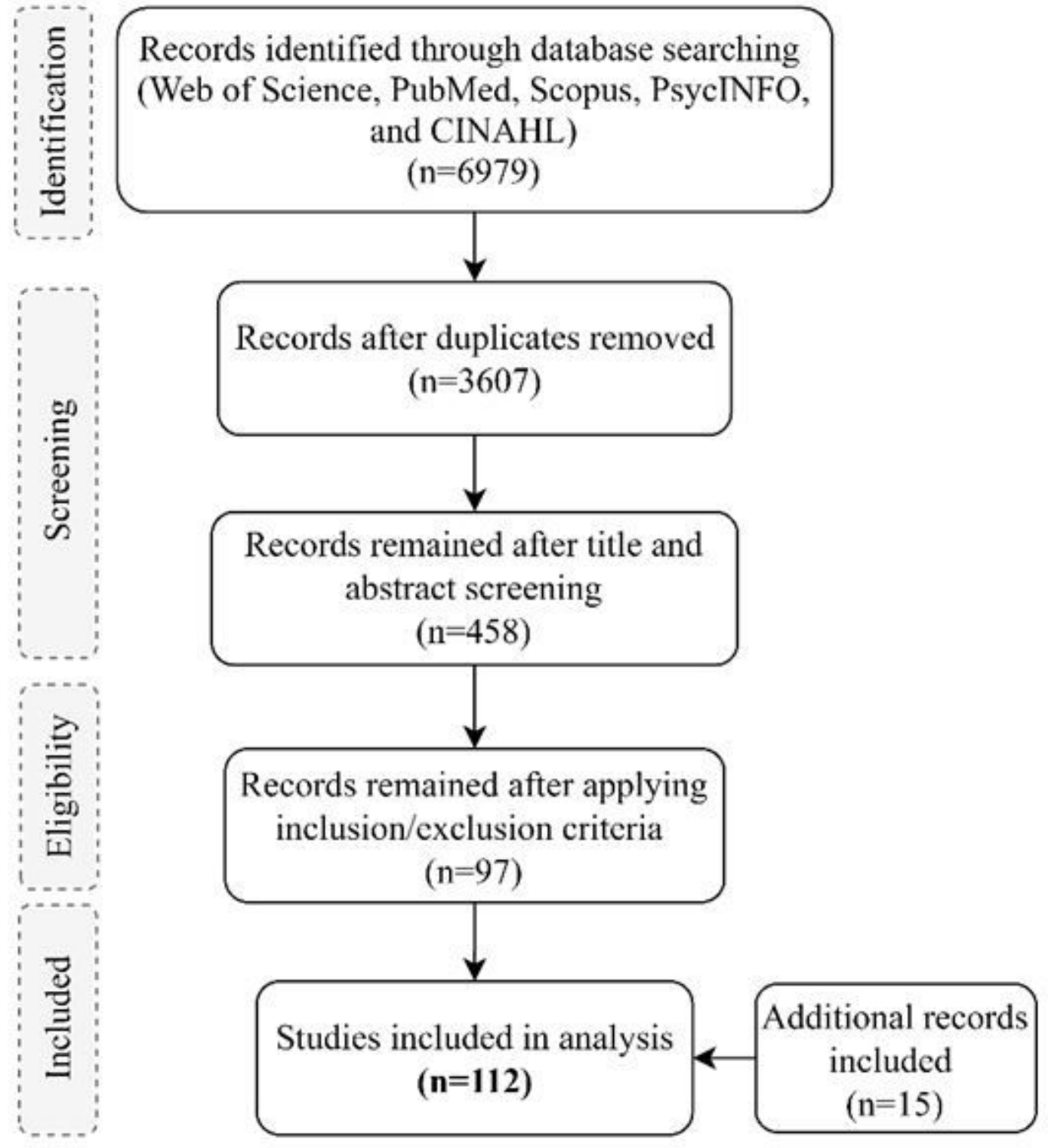

Figure 1

Literature searching flow chart 


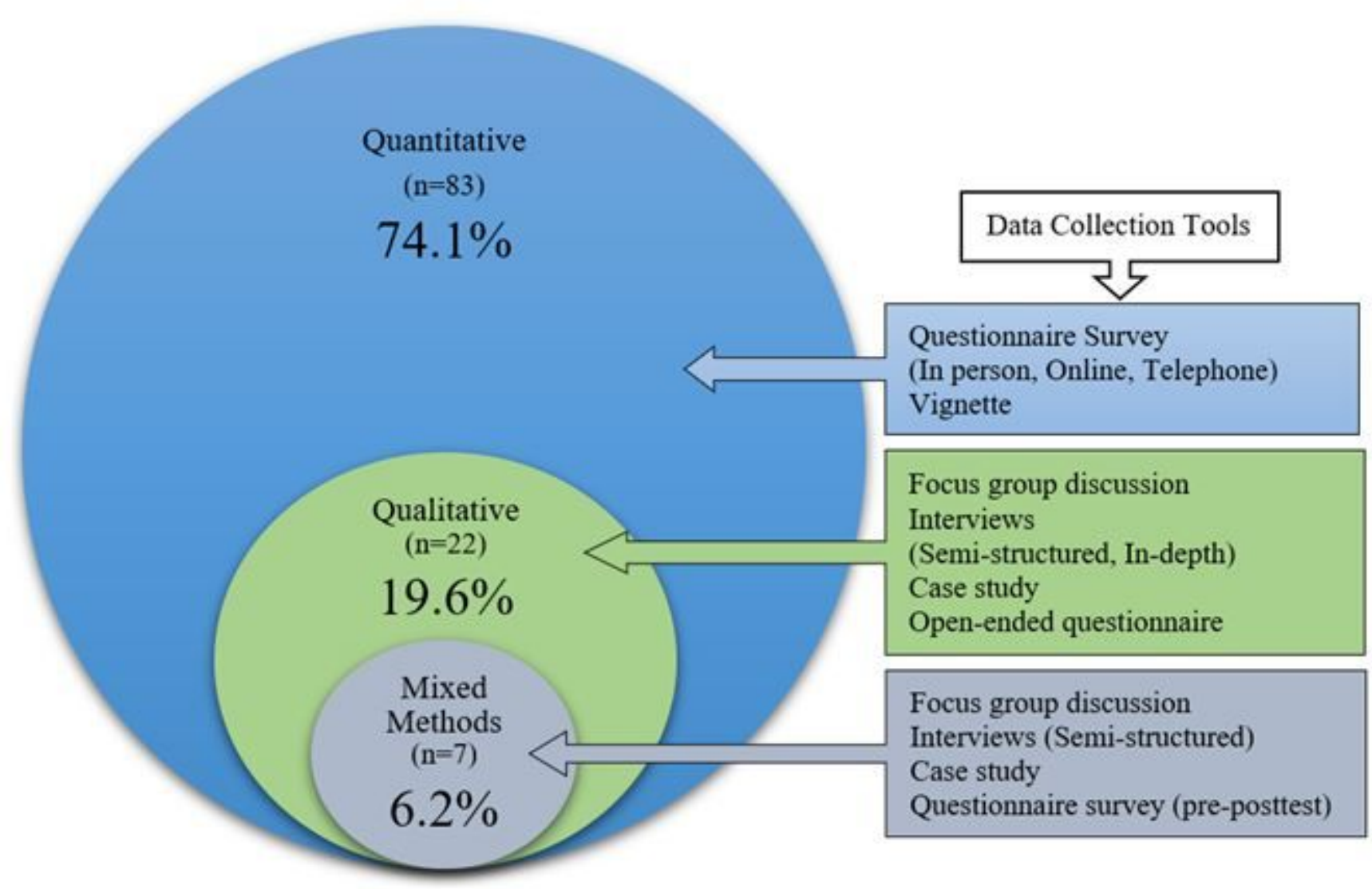

Figure 2

Study design and data collection tools

\section{Supplementary Files}

This is a list of supplementary files associated with this preprint. Click to download.

- SupplimentaryFile.docx

- SupplementaryTableA.docx 